\title{
Energy efficient geographical and power based clustering algorithm for heterogeneous wireless sensor networks
}

\begin{abstract}
Wireless Sensor Network (WSN) has gained tremendous research attention recently due to their extensive applications. As sensor nodes being battery operated, many researchers have made attempts to prolong the lifespan of the WSN by reducing the-per node energy consumption and efficiently utilizing the sensor nodes. However, in the tradition WSNs, nodes were homogeneous and hence could not take full advantage of the presence of heterogeneity in the network. To solve this problem in this paper, we propose Geographical and power based clustering algorithm (GPCA): a heterogeneous-aware clustering protocol, which has significant impact on the entire energy dissipation of WSNs. In GPCA, a Virtual Header $(\mathrm{VH})$ transfers data to the nearest $\mathrm{VH}$, and the nearest $\mathrm{VH}$ forwards the data to sink node. In this way, the energy dissipation of the entire network is reduced because of the transmitting distance between VHs and the sink that is greatly shortened. Also, a large number of nodes are self-organized by a distributed cluster formation technique. Moreover, a randomized technique is used to rotate the local cluster-heads base on power label in order to evenly distribute the energy load among the sensors in the network. GPCA uses geographical position to enable scalability and robustness for dynamic networks. By using simulation, the proposed GPCA scheme shows superior performance over the current energy-efficient schemes in terms of network lifespan, Energy dissipation and number of alive nodes.
\end{abstract}

Keyword: Energy efficiency of WSN; Game theory; GT DSA MAC; MAC; WSN 